\title{
NETest: A Systematic Review Focusing on the Prognostic and Predictive Role
}

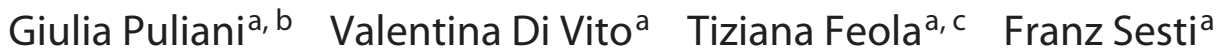 \\ Roberta Centello ${ }^{a}$ Carla Pandozzi $^{a}$ Maria Grazia Tarsitano ${ }^{a}$ Monica Verrico $^{d}$ \\ Andrea Lenzi $^{a}$ Andrea M. Isidori ${ }^{a}$ Elisa Giannetta ${ }^{a}$ Antongiulio Faggiano ${ }$ \\ aDepartment of Experimental Medicine, Sapienza University of Rome, Rome, Italy; ${ }^{\mathrm{b} O n c o l o g i c a l ~ E n d o c r i n o l o g y ~ U n i t, ~}$ \\ IRCCS Regina Elena National Cancer institute, Rome, Italy; 'Neuroendocrinology, Neuromed Institute, IRCCS, Pozzilli,

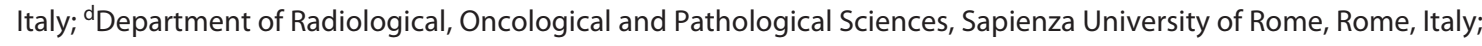 \\ eEndocrinology Unit, Department of Clinical and Molecular Medicine, Sant'Andrea Hospital, Sapienza University of \\ Rome, Rome, Italy
}

\section{Keywords}

NETest · Neuroendocrine neoplasms · Liquid biopsy ·

Predictive biomarker · Prognostic biomarker

\begin{abstract}
The NETest is a standardized and reproducible liquid biopsy for neuroendocrine tumors (NETs). It evaluates the expression of 51 NET genes by real-time polymerase chain reaction, providing an accurate molecular profile of the neoplasm. Diagnostic utility of NETest has been widely demonstrated, while its role in predicting prognosis and treatment response is less studied. This systematic review aims to collect and discuss the available evidence on the prognostic and predictive role of NETest, trying to answer 3 questions, frequently raised in clinical practice. Is NETest able to differentiate stable from progressive disease? Increased NETest levels (at least $>40 \%$ ) correlate with disease progression. Is NETest able to predict tumor progression and tumor response to treatment? Some studies demonstrated that the baseline NETest score $>33-40 \%$ could predict tumor progression. Moreover, NETest performed after treatment (as peptide receptor radionuclide therapy) could predict treatment response also be-
\end{abstract}

fore radiological findings, since the decrease or stability of NETest score predicts tumor response to treatment. Is NETest able to evaluate tumor recurrence risk after surgery? NETest can predict surgical treatment outcome detecting minimal residual disease after radical surgery, which is characterized by a lower but positive NETest score (20-40\%), while a higher score (>33-40\%) is associated with nonradical surgery. In conclusion, in addition to its demonstrated diagnostic role, this systematic review highlights the efficacy of NETest to assess disease status at the moment of the NETest execution and to predict tumor recurrence after surgery. The efficacy for other applications should be proven by additional studies.

(c) 2021 S. Karger AG, Basel

\section{Introduction}

Neuroendocrine neoplasms (NENs) are heterogeneous in terms of primary sites, neuroendocrine differentiation, clinical behavior, and response to treatments. In this field, the possibility to relay on easy to execute markers for estimating the prognosis and for predicting re- 
sponse to treatment could be essential for improving the clinical management of these patients [1]. Nowadays in patients affected by neuroendocrine tumors (NETs), the strongest predictors of overall survival (OS) are tumor grade and stage [2].

Circulating biomarkers have been studied for diagnosis and follow-up of patients affected by NENs. While in functional NENs it is possible to analyze secretory products or their metabolites in blood and/or in urinary sample, in nonfunctioning tumors only the so-called general tumor markers can be used [3]. Chromogranin A ( CgA) is the most used general tumor marker, with both diagnostic and predictive value [4]. The prognostic role of this marker has been advocated since the demonstration of the correlation with OS [5], poorer outcome [6], and tumor burden [7]. However, there are multiple limitations of CgA use. A false-positive value can be observed in nononcological diseases, such as atrophic gastritis, hypergastrinemia, heterophile antibodies, impaired kidney function, or use of antisecretory medications, especially proton pump inhibitors $[3,8,9]$. False-negative results can happen in case of less differentiated disease, since CgA is a secretory product of the neuroendocrine cells [10].

Neuron-specific enolase (NSE) is physiologically present in neurons and neuroendocrine cells and its circulating form is a biomarker for OS in gastroenteropancreatic (GEP) NEN patients $[11,12]$. However, serum NSE is increased only in $30-50 \%$ of these patients [13]. A significant association of NSE levels with survival was also demonstrated in patients affected by small cell lung cancer [14]. As a prognostic marker of NETs, NSE is minimally correlated with tumor size but associated with grading. In fact, its levels result higher in patients with poorly differentiated neuroendocrine carcinoma (NEC) [15].

Preliminary data of the use of serum neutrophil-lymphocyte ratio (NLR) are available in patients affected by lung NECs. In these patients, an increasing preoperative NLR is associated with higher stage and inversely correlates with post-resection OS and relapse-free survival [16, 17]. The predictive value of worse survival of the pre-operative NLR was also demonstrated in patients with gastric NENs undergoing surgery [18] and in intestinal and pancreatic NETs $(\mathrm{Ki}-67<10 \%)$ treated with lanreotide [19]. Recently, NETest has been developed and proposed mostly for the diagnosis of NENs, demonstrating in a recent meta-analysis on 10 studies a diagnostic accuracy of 95-96\% [20].

The aim of this systematic review is to collect and discuss the available evidence on the role of NETest in predicting prognosis and treatment response, including both systemic treatments, used in metastatic patients, and surgical and ablative strategies, used in localized disease, in patients affected by NENs, trying to answer the following questions: (1) Is NETest able to differentiate stable from progressive disease? (2) Is NETest able to predict tumor progression and response to therapy? (3) Is NETest able to predict tumor recurrence after surgery?

\section{Materials and Methods}

We performed this study according to the Cochrane Collaboration and PRISMA statement [21].

\section{Data Sources and Searches}

From June to November 2020, we searched for English-language articles in MEDLINE. No date restriction has been applied. Search terms used were: "NETest"; "predictive biomarker" AND "neuroendocrine"; "prognostic biomarker" AND "neuroendocrine"; "liquid biopsy" AND "neuroendocrine." Additionally, we searched in EMBASE, Cochrane Library, and SCOPUS using "NETest" as a search term.

Eligibility criteria for study selection included studies on humans with any of the following designs: randomized clinical trials, prospective non-randomized trials, retrospective studies, and case series. We selected: (1) articles on NETest; (2) data on prognostic value or treatment response prediction or treatment response assessment of NETest; and (3) patients affected by any subtypes of NENs. Exclusion criteria were: (1) non-original articles or case reports; and (2) articles reporting only data on the diagnostic value of NETest. A final update of the search was conducted in May 2021 and one additional study was included.

\section{Article Selection}

Each study was screened by abstract and title and potentially eligible studies were further assessed in detail by retrieving fulllength articles. Each full-length article was independently reviewed by two separate authors (G.P. and V.D.V.) following inclusion criteria. Two authors independently extracted data from the articles that met the inclusion criteria. A standardized form was used to extract the following information: year of publication, type of study, number of included patients, age at diagnosis, sex, histopathological examination, staging and outcomes, treatment strategy (surgery, medical treatment, and radiotherapy), time of execution and values of NETest, correlation of NETest with disease status, prognosis, and treatment response. Quality of studies has been assessed by MINORS score [22].

\section{Results}

From the original number of 244 articles, 26 have been selected by title and abstract. After full-text evaluation, a total of 20 articles were included in the systematic review (Fig. 1). 
Fig. 1. Flow diagram of included and excluded studies. *Respectively on PubMed, Embase, Cochrane Library, and Scopus. No time restriction has been applied in the search strategy; articles included in the systematic review have been published from 2015 to 2021.

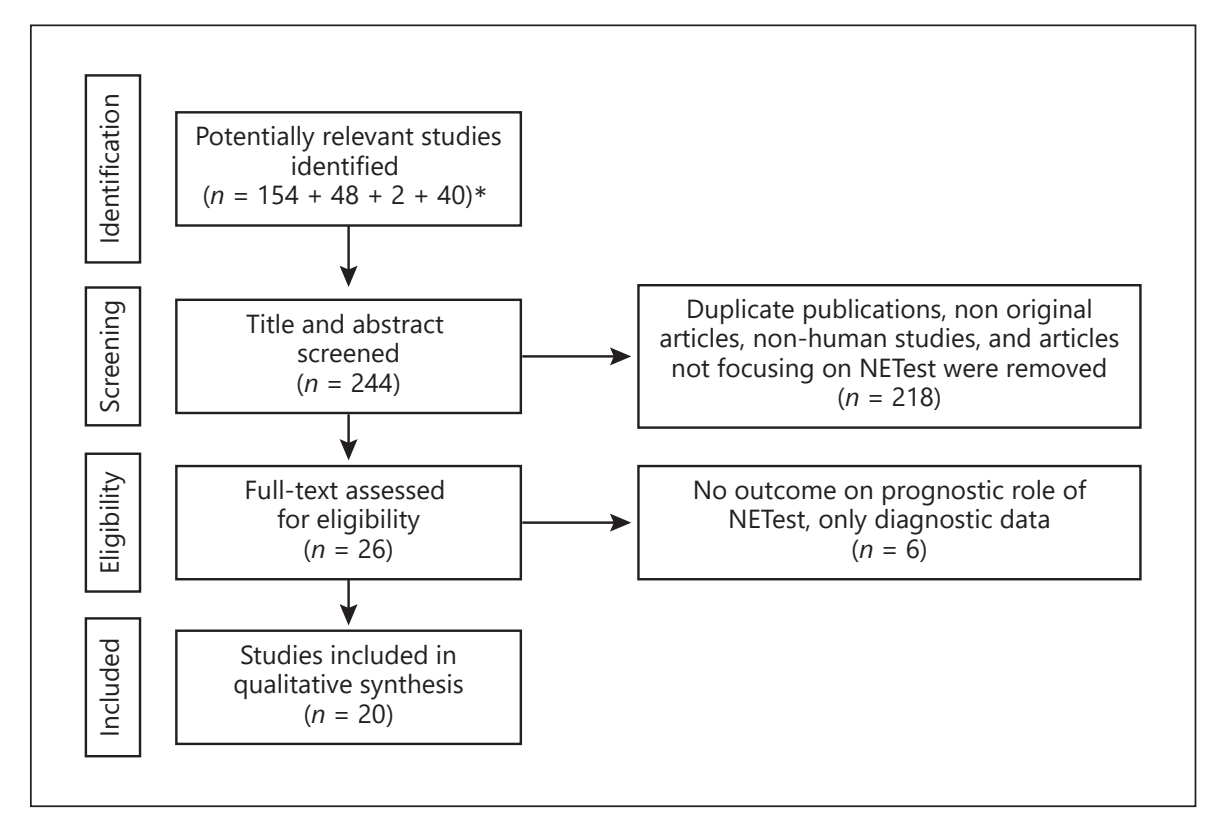

\section{Liquid Biopsy and NETest}

In recent years, liquid biopsy has received growing attention. It is a molecular biology technique that allows to identify and to characterize neoplasms, through molecular analyses performed on a venous blood sample. Liquid biopsy is performed analyzing the following elements: circulating cancer cells, free nucleic acids, exosomes, and tumor-educated platelets (TEPs); the name of the latter originates from their ability to engulf the circulating RNA released by the tumor, with therefore a correlation between TEPs and tumor growth and dissemination in various kinds of neoplasms $[23,24]$. One of the advantages of liquid biopsy analysis is that it can be serially repeated, allowing to get real-time information from the lesion so that we can promptly make changes in therapy [25]; moreover, given the characteristic heterogeneity of NENs, this diagnostic strategy could probably be more effective in representing the totality of the disease compared to a biopsy that could only provide a partial view $[26,27]$.

In this field of liquid biopsy applied to NENs, NETest has been developed in recent years. This is a standardized and reproducible clinical laboratory measurement for the diagnosis of NENs, whose clinical utility has been documented in GEP and bronchopulmonary NENs and in paragangliomas and pheochromocytomas (PPGLs) [2831]. The efficacy of this biomarker for diagnostic purpose originates also from its independence from patient's characteristics, such as age, sex or ethnicity, and treatments. As a result, it is advantageous compared to CgA $[31,32]$.
After mRNA isolation and cDNA production, NETest uses real-time polymerase chain reaction (RT-PCR) in order to quantify circulating transcriptional products of neoplastic origin. In particular, NETest evaluates the expression of 51 genes, 30 of which can be categorized into 9 clusters, related to cell proliferation and apoptosis (Proliferome, Growth factor signalome, and Apoptoma), peptide secretion (Secretome I, general and Secretome II, progressive), epigenomic changes (Epigenome), and somatostatin receptor (SSTRome), providing tumor molecular profile $[33,34]$. After RT-PCR, results are analyzed through algorithms, designed to differentiate healthy controls from NENs patients, determining a 0-8 score: samples scored $0-2$ are classified as normal, while levels 3-8 are categorized as NENs [35]. To expand the utility of the NETest from a diagnostic tool to an instrument able to capture the biology of NENs, a second algorithmbased analysis quantifies the expression of 6 of the abovementioned clusters (SSTRome, Proliferome, Metabolome, Secretome, Epigenome, and Plurome) and, incorporated also the machine-learning derived 0-8 score, generates a clinical activity score scaled from 0 to $100 \%$ (the NETest score). While the first score is able to diagnose NENs from controls, the second score is related to tumor activity, being more able to differentiate stable (SD) from progressive disease (PD) [31]. Thus, the NETest is able to capture the biology of a specific NEN defining its molecular status. 

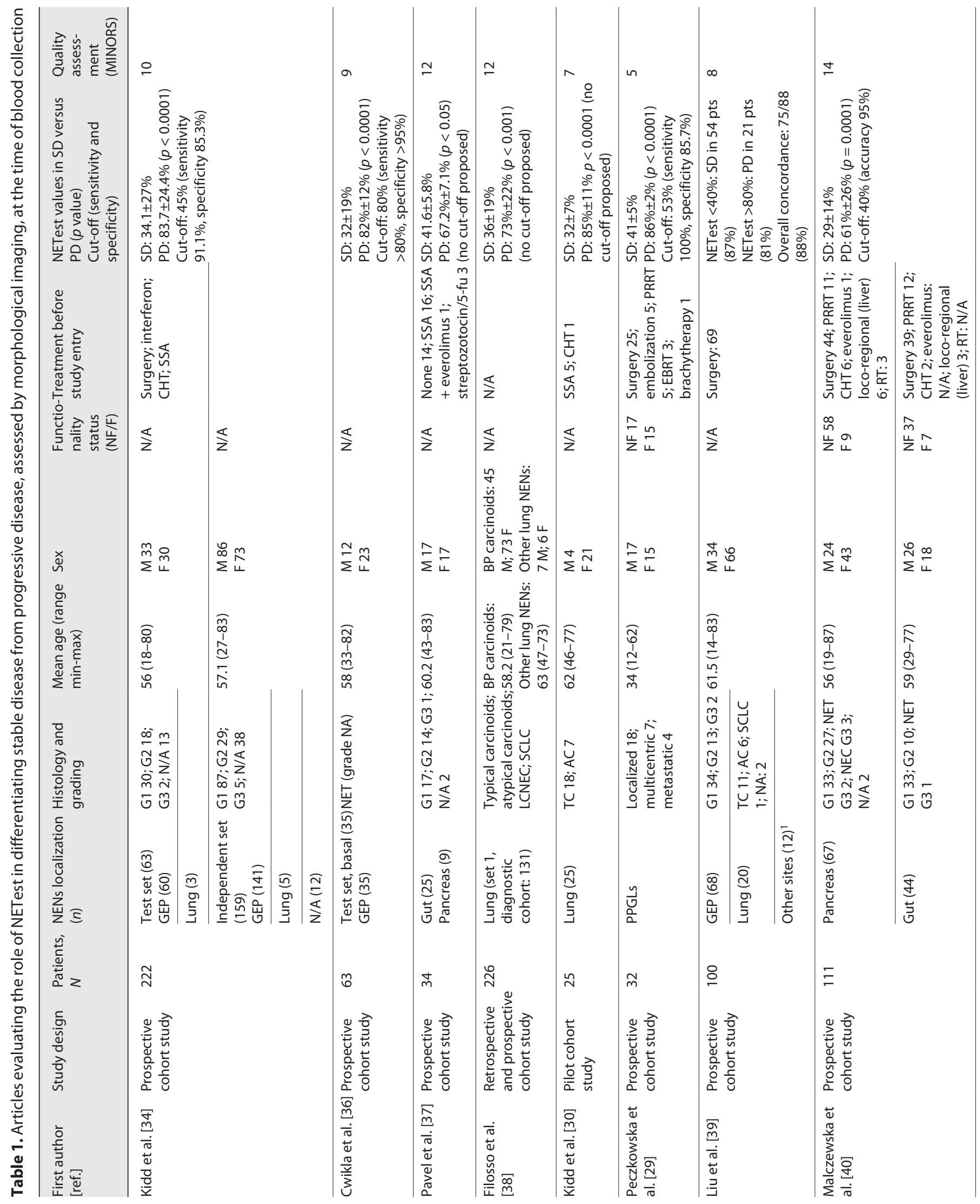


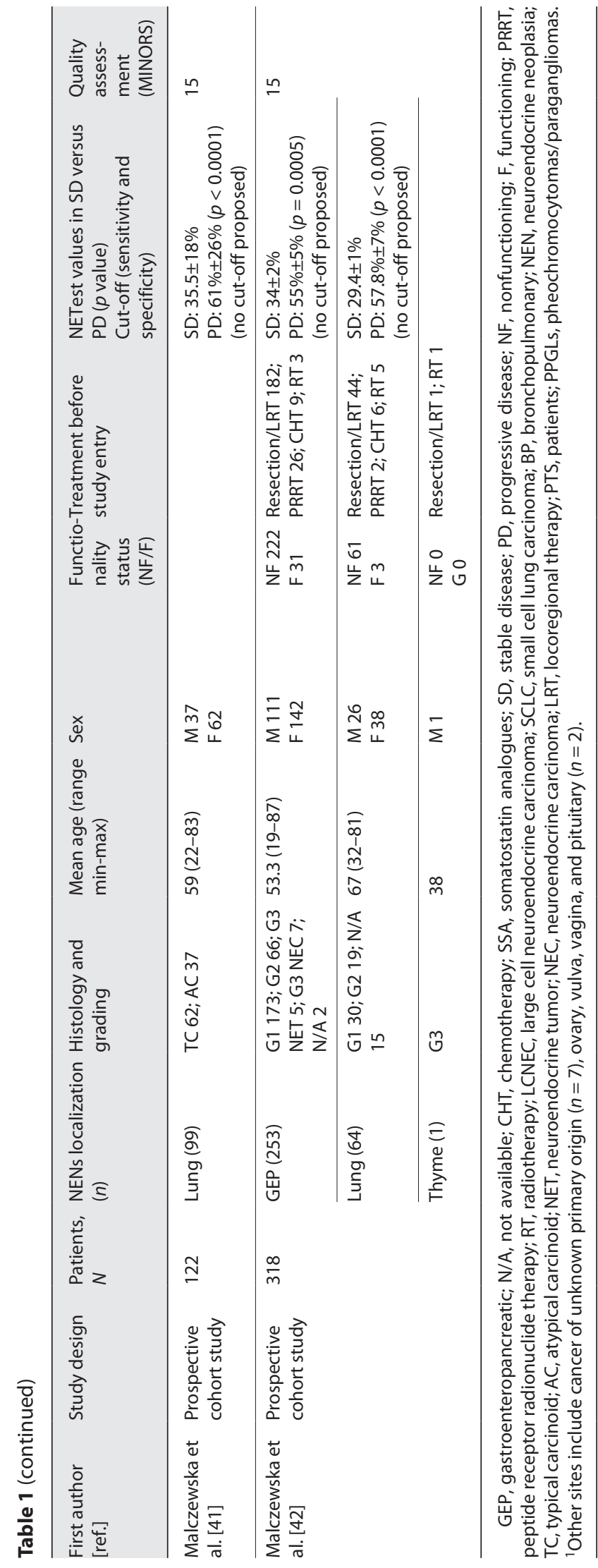

\section{Prognostic Role of NETest}

Is NETest Able to Differentiate Stable from

Progressive Disease?

Ten studies have demonstrated that NETest is able to differentiate between SD and PD, in many subtypes of NENs (Table 1). Using the initial linear score, ranging for $0-8$, Kidd et al. [34] demonstrated that a score between 0 and 5 was associated with SD in over $90 \%$ of patients, while a score of 8 correctly identified PD in over $90 \%$ of patients. The study was performed on an overall population of 111 patients with SD and 48 with $\mathrm{PD}$, mostly grade 1-2 GEP-NET [34]. In the same study, using the NETest score, the authors demonstrated that SD samples had significantly lower activity scores than PD samples (34.1 \pm $27 \%$ vs. $83.7 \pm 24.4 \%, p<0.0001)$, proposing $45 \%$ as a cutoff (upper 95\% confidence interval for SD) [34].

Malczewska et al. [40], in a study on 72 patients affected by radiologically detectable GEP-NENs (42 pancreatic NETs, 33 small-intestinal NETs), 11 progressive and 64 stable, confirmed that the NETest score was significantly increased in PD (mean \pm standard deviation: 61 $\pm 26 \%$ ) compared to SD (29 $\pm 14 \%)$; the cutoff score of $40 \%$ showed an accuracy of $95 \%$. Similar results were also confirmed by Pavel et al. [37] (PD group vs. SD group $67.2 \pm 7.1 \%$ vs. $41.6 \pm 5.8 \%, p<0.05)$.

The ability of NETest to differentiated PD from SD was confirmed also in studies enrolling only pulmonary NENs. In a study on 112 patients, the NETest score was significantly lower in SD $(36 \pm 19 \%)$ than in PD $(73 \pm$ $22 \%, p<0.001$ ) [38]. In a cohort of 25 typical and atypical lung carcinoids, NETest was confirmed to differentiate tumor progression ( $85 \pm 11 \%$ vs. $32 \pm 7 \%$ ) [30]. Despite the good performance, as demonstrated by high AUC, in both cases the authors did not propose a specific cutoff $[30,38]$. Another study confirmed that NETest accurately distinguished PD from SD (61 \pm 26 vs. $35.5 \pm 18$, $p<$ 0.0001 ); moreover, in pulmonary carcinoids (data on 99 patients), NETest levels were elevated in metastatic disease irrespective of histology (typical, 78 patients, and atypical carcinoids, 21 patients), both in SD cohort and in PD cohort [41]. A recent study by Malczewska et al. [42] confirmed, on a large series of patients (135 GEP and 64 pulmonary), that NETest was able to differentiate $\mathrm{PD}$ from SD both in GEP-NENs ( $55 \pm 5$ vs. $34 \pm 2, p=0.0005$ ) and in pulmonary carcinoids (57.8 \pm 7 vs. $29.4 \pm 1, p<$ 0.001) and PPGLs [29].

However, other articles identified a cutoff of $80 \%$ for PD [36]. This higher cutoff ( $80 \%)$ has been identified also in the study by Liu et al. [39]. The study demonstrated the high concordance between low NETest $(<40 \%)$ and SD 
(83\% of patients with SD) and high NETest $(>80 \%)$ and PD (60\% of patients with PD), with an overall concordance of $88 \%$. Other studies demonstrated that a significant difference in NETest score is also found between localized and metastatic disease, both in GEP and in pulmonary NENs $[42,43]$.

A study on patients affected by PPGLs identified a cutoff of 53\% for differentiating PD (11 patients) and SD (19 patients) at the moment of the blood collection (0.93, confidence interval: $0.84-1.03, p<0.0001$ ); moreover, the NETest score was significantly higher in multicentric and metastatic disease than in localized disease [29].

In summary, the NETest score $>40 \%$ demonstrated a high concordance with progression evidenced by radiological imaging. This finding does not change also considering prospective studies only. This is in accordance with the cutoff of $40 \%$ reported in the meta-analysis by Oberg et al. [20].

Is NETest Able to Predict Tumor Progression and Tumor Response to Treatment?

Some studies have evaluated the possibility for NETest to predict tumor progression in the months following the execution of the NETest (Table 2). An interesting study by Pavel et al. [37], on 34 patients affected by stable GEPNETs, demonstrated that, in patients with SD at baseline, the basal value of NETest $>40 \%$ can predict subsequent tumor progression despite multimodal treatment strategies, while values $<40 \%$ predict stability over 5 years. Moreover, the basal NETest score was associated with progression-free survival (PFS) (hazard ratio $=1.022$, $95 \%$ confidence interval $=1.005-1.04, p<0.012)$ and Kaplan-Meier analyses demonstrated that baseline NETest $<40 \%$ predicted longer PFS (median PFS 2.78 years in case of basal NETest $<40 \%$ and 0.68 years in case of basal NETest $>80 \%$ ) [37].

The correlation of basal NETest score with PFS was confirmed also in a cohort of 100 patients (68 GEP, 20 broncopulmonary, and 12 mixed NENs). Mean PFS of patients with NETest scores $<40 \%$ without treatment was 12 months, while mean PFS was only 3 months in the case of basal NETest scores $>80 \%$, despite treatment [39]. In the same study, Liu et al. [39] evaluated the utility of the NETest in a watch-and-wait program (45 patients). Patients with a basal low score $(\leq 40 \% ; n=27)$ maintained $\mathrm{SD}$, while all patients with a high NETest $(\geq 80 \% ; n=14)$ required treatment intervention and/or developed $\mathrm{PD}$ [39].

A recent interesting prospective study has enrolled 152 patients with sporadic GEP-NENs, followed for 36 months (range 4-56); 119 had measurable disease and 33 had no evidence of disease at enrolling. Basal NETest categories (low tumor activity $<33 \%$; intermediate tumor activity $34-79 \%$; and high tumor activity $\geq 80 \%$ ) predict median PFS, which was, respectively, 55, 18, and 11 months. Patients with NETest $>33 \%$ had an overall 9 times higher risk of developing PD than those with NETest $\leq 33 \%$ (with a reported odds ratio of 8.6). Of the overall number of 152 patients, 55 with measurable disease were enrolled in the watchful waiting group (no treatment) and $32 \%$ of these patients developed PD within 1 year. Only $16 \%$ of patients with low tumor activity had PD, compared to $50 \%$ and $54 \%$ of intermediate and high activity categories. In parallel, 64 patients were treated since baseline and PD within 12 months of follow-up were observed in $45 \%$. Once again, basal NETest predicted the risk of PD at 12 months: progression was observed in $17 \%$ of patients with low activity scores, in $61 \%$ with intermediate, and in $74 \%$ with high tumor activity. As confirmation, $70 \%$ of patients with low tumor activity in the watchful waiting group and $64 \%$ of patients with low tumor activity in the treatment group had SD after 24 months. Finally, considering the 33 patients without evidence of disease at baseline, no patients with negative NETest $(<20 \%)$ developed recurrence; moreover, the median value of NETest in patients who remain free of disease at follow-up was $27 \%$ compared to $53 \%$ in patients with recurrence [45].

Cwikla et al. [36], in the prospective part of their study, evaluated NETest score before starting somatostatin analog (SSA) treatment for GEP-NETs (G1 and G2). A basal level of $80 \%$ predicts the development of disease progression in $100 \%$ of patients (14 patients). Even in case of basal NETest $<80 \%$, the NETest score was higher in patients $(57.5 \pm 6 \%$ vs. $41 \pm 2 \%, p=0.02)$ that subsequently developed PD [36].

Some studies, reporting multiple NETest determinations, evaluated if NETest score variation could assess treatment efficacy. These studies focused mainly on peptide receptor radionuclide therapy (PRRT) and SSA.

Bodei et al. [44] evaluated the changing in NETest score according to PRRT efficacy. In this study, responders to PRRT were defined as patients showing complete response, partial response, or SD evaluated by computed tomography according to Response Evaluation Criteria In Solid Tumors (RECIST) version 1.1, while non-responders showed tumor progression. After 6 months from treatment starting, a reduction in NETest was seen in $88 \%$ of responder patients (no change in $12 \%$ of responders), while an increase in NETest was seen in $90 \%$ of non-responders, showing high agreement between ra- 
diological findings and NETest (89\% of concordance) [44]. These preliminary results have been subsequently confirmed by another study on 122 patients affected by lung and GEP-NETs. In case of objective response to PRRT, NETest decreased (mean change: $-47 \pm 3 \%$ ), while NETest remains high in the majority of non-responders. Authors proposed a post-treatment cutoff of $40 \%$ for differentiating SD from PD and therefore for determining PRRT response [46].

In a study on repeated NETest measurements on 9 patients affected by PPGLs, 4 patients with SD at baseline and a good response to treatment with SSA demonstrated a fall or a stability in the NETest score at follow-up, while $2 \mathrm{SD}$ at baseline with following PD showed an increase in NETest score. Considering patients with PD at baseline, the NETest score increases in 1 patient with further progression, decreases in 1 patient who underwent surgery, and remains stable in 1 patient without further progression on SSA treatment [29]. Cwikla et al. [36] demonstrated that elevated NETest (80-100\%) during SSA treatment for GEP-NETs (G1 and G2) was significantly associated with therapy failure (sensitivity $100 \%$; specificity $57 \%$; positive predictive value $70 \%$; and negative predictive value $100 \%$ ).

Liu et al. [39], in a cohort of 55 NEN patients treated mainly with SSA (84\%), confirmed that NETest was clinically useful in treatment monitoring. In fact, $100 \%$ of patients with a low score exhibited SD at 6-12 months, while an increase in NETest during treatment required further therapy modifications, as an increase in SSA dosage or addition of an alternative agent [39].

Overall, high NETest values $(>40 \%)$ in patients without contemporary radiological progression could predict subsequent progression. All the available studies are prospective, but since study treatments and end-points vary between studies, no definitive conclusions on the application of NETest in this clinical context can be deduced.

Is NETest Able to Evaluate Tumor Recurrence Risk after Surgery?

In a study on 35 GEP-NENs (only 1 G3), in which 27 patients underwent surgical resection and 8 embolization [35], resection was associated to a decrease in the NETest score (from $80 \pm 5 \%$ to $29 \pm 5 \%, p<0.0001$ ). The study included NETest evaluation 1 month after treatment and radiological evaluation 3 and 6 months after treatment. Interestingly, considering the surgical cohort, authors compared $15 \mathrm{R} 0$ patients with $12 \mathrm{R} 1$ patients. In the group with radical surgical resection, the reduction in NETest score was more marked (R0: from $80 \pm 6.3 \%$ to $28.9 \pm$
5.5\%; R1: $79.5 \pm 8.5 \%$ to $47.2 \pm 9.9 \%)$. Moreover, the same authors reported that 12 patients with $\mathrm{R} 0$ and no clinical or radiologic evidence of disease recurrence after 5 years showed a maximum post-operative NETest score of $14 \%$ (negative score), while in other cases the NETest score showed a moderate disease activity ( $>20 \%)$. This is consistent with the finding that 4 of $11 \mathrm{R} 0$ patients with increased NETest at 1 month developed subsequent positive imaging (sensitivity 100\%, specificity 20\%) [35].

In a study on 13 small intestine NETs, surgery determined a significant lowering of NETest score. However, a significant reduction was associated to curative surgery (NETest $20-40 \%$, classified as low), while, in case of resection in the setting of metastatic disease, the reduction was lower; in particular in case of PD after surgery, the NETest score was above the levels of $40 \%$ (over $60 \%$ in $3 / 5$ cases) [47]. Similarly, in a study reporting NETest score before and 6 months after a combined treatment with both surgical intervention and PRRT in 9 patients affected by metastatic small intestinal NETs, authors reported a reduction in NETest score after treatment (from $83 \pm$ $12 \%$ to $34 \pm 15 \%)$ and identified $40 \%$ as post-treatment cut-off for detecting SD [48].

Genc et al. [49] analyzed the role of NETest, performed after surgery, in predicting recurrence of pancreatic NETs (G1 and G2), identifying a cut-off of $40 \%$ (false-positive or false-negative patients were $18 \%$ ). Interestingly, NETest was higher in patients with recurrence (R0 at histology) than in patients diagnosed as R1, but without clinical recurrence [49].

Partelli et al. [50] evaluated NETest before and after surgery on 30 patients affected by pancreatic NENs. Beyond the efficacy of NETest in diagnosing NETs before surgery, after surgery all patients demonstrated a decrease in NETest levels without differences in each time evaluated (post-operative day, POD 1, 5, and 30). Interestingly, among 3 patients showing levels of NETest $>40 \%, 2$ of them had R1 resection, and 1 had potentially nodal involvement. Among the remaining 15 patients, 12 exhibited a mean NETest level of $27 \%$ after resection (POD30), which is consistent with the presence of residual disease; even if the follow-up is not enough for identify recurrence, it is possible to hypothesize that the NETest score is able to identify patients at risk for recurrence [50]. In another study on gastric NENs, 5 patients underwent total gastrectomy and 8 subjects underwent partial gastrectomy. Despite all patients were disease free at endoscopical, radiological, and functional imaging, the NETest score was elevated in 6 patients, suggesting that these patients could have minimal residual disease. According 


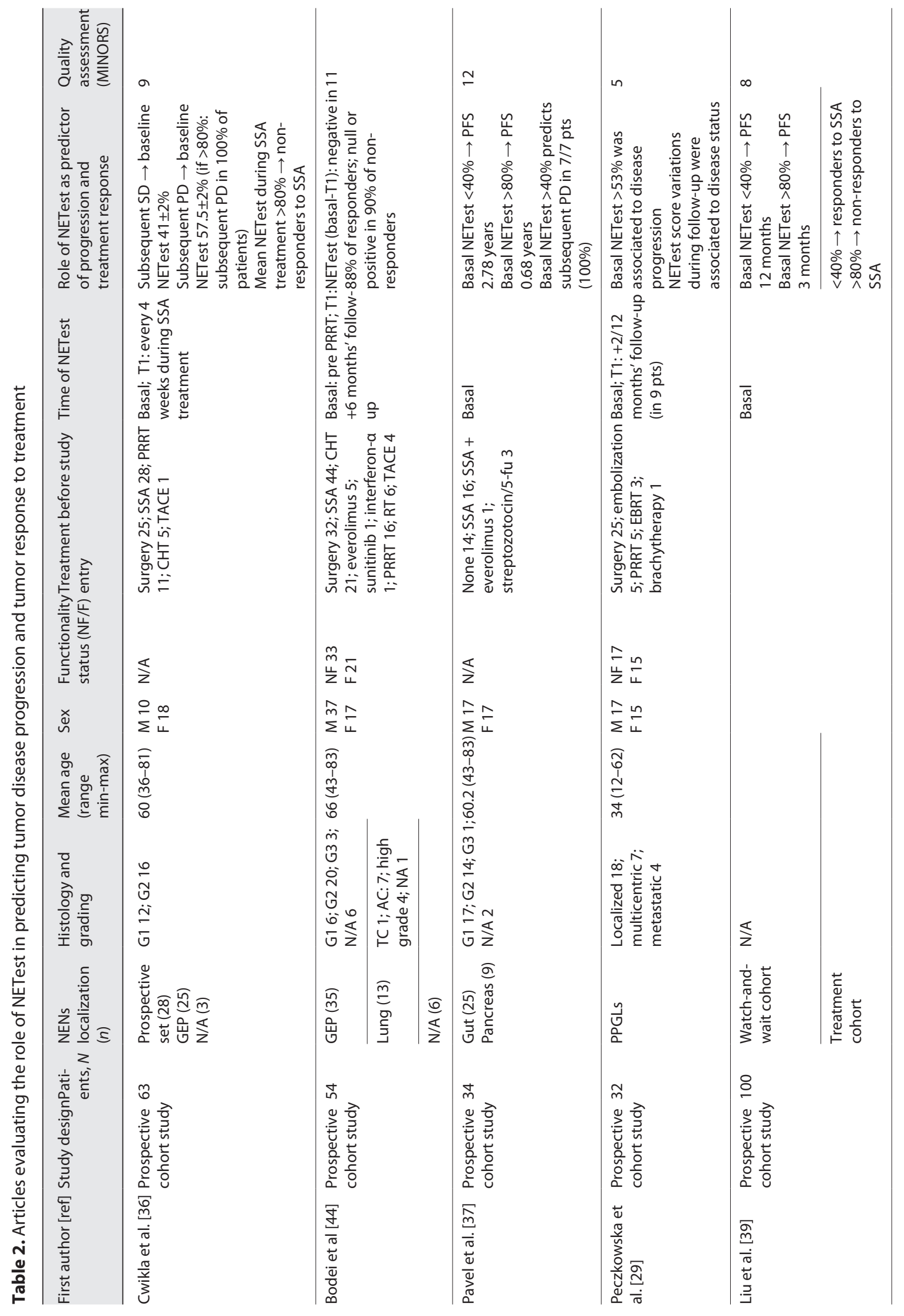




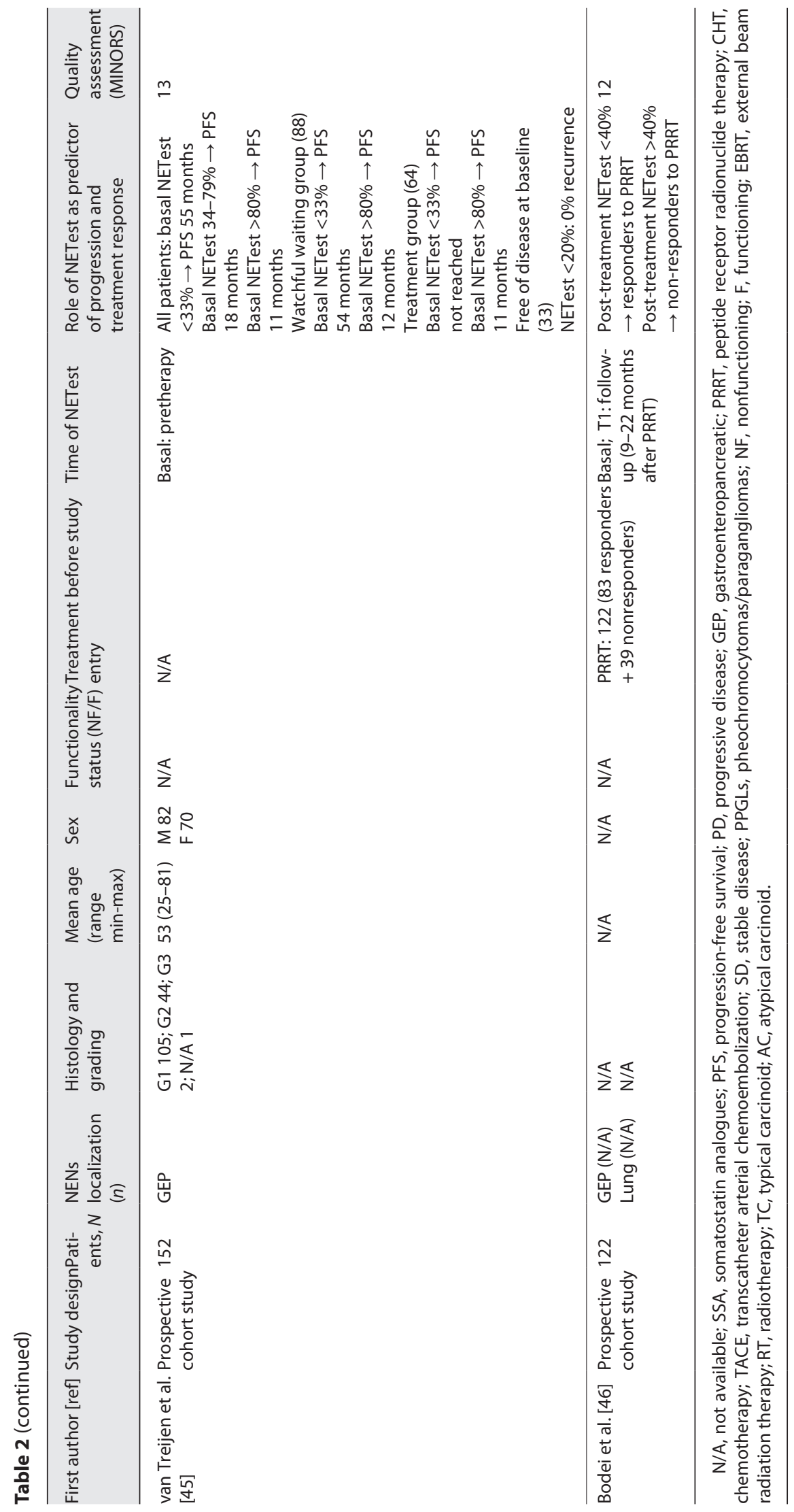




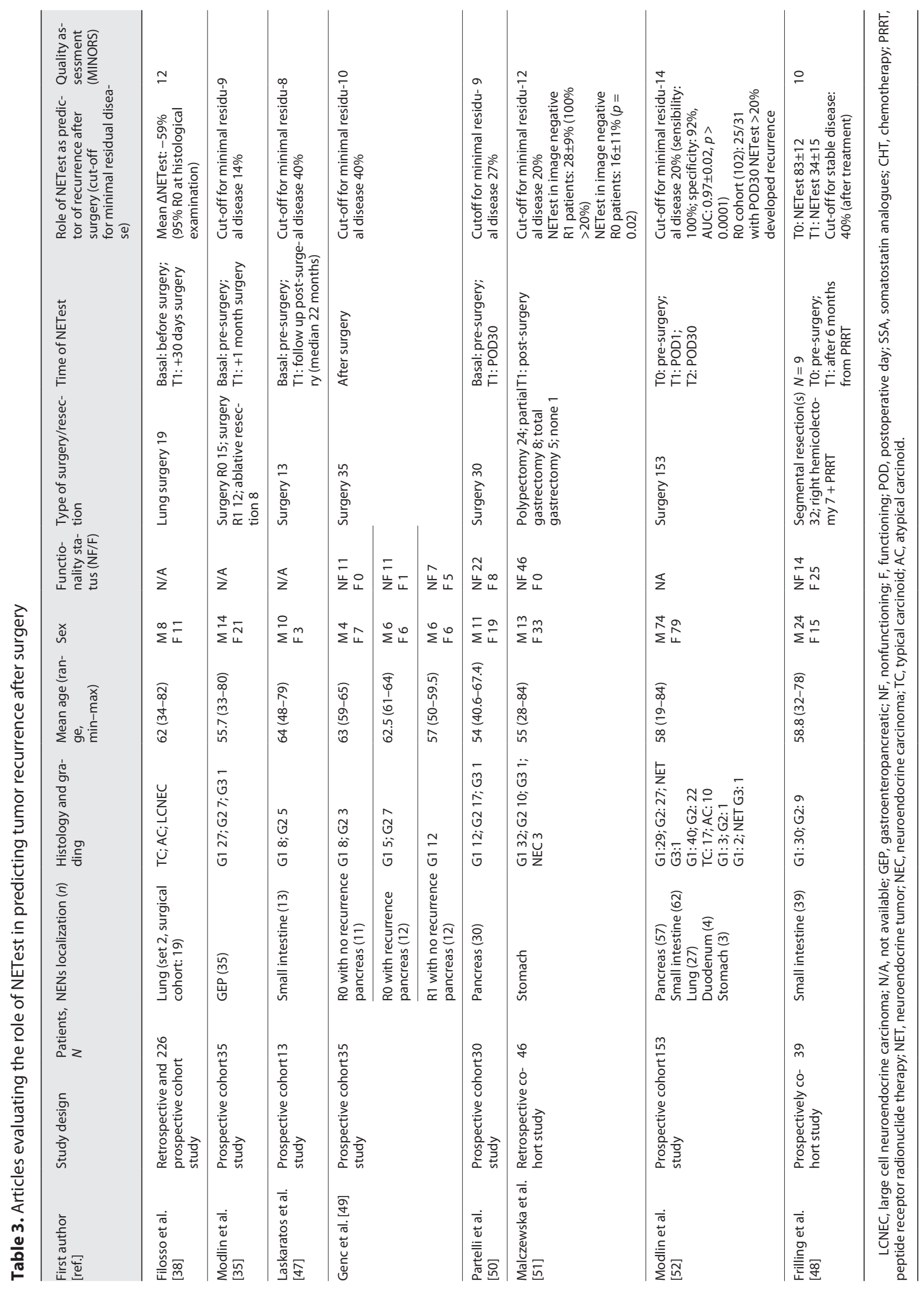


to this hypothesis, the same study demonstrated that in case of microscopical residual disease evidenced at histological examinations ( 5 patients with positive polypectomy margin and 4 patients with random biopsies diagnostic for microscopic tumor), NETest was elevated (28 \pm $9 \%)$, demonstrating the accuracy of this diagnostic instrument in detecting minimal residual disease before morpho-functional imaging [51].

In a recent study, Modlin et al. [52] evaluated NETest pre-operatively, on POD1 and POD30 in a cohort of 153 patients affected by surgically treated pulmonary and GEP NETs. In the R0 cohort, POD30 NETest levels decreased but remained elevated $(<20 \%)$ in 31 patients, and $25 / 31$ patients with a POD30 NETest $>20 \%$ developed image-identifiable recurrence by the following 18 months. However, all the patients with POD30 NETest values $<20 \%$ were free from recurrence during follow-up. The authors demonstrated that a NETest value $>20 \%$ on POD30 predicted residual disease with $94 \%$ accuracy and $100 \%$ sensitivity [52].

Finally, the role of NETest in evaluating the efficacy of surgical excision has been confirmed also in pulmonary NEN. In the study by Filosso et al. [38], in a subgroup of 19 patients with pulmonary NEN (12 typical carcinoids, 4 atypical carcinoids, and 3 large-cell NECs), the NETest score significantly decreased 30 days after surgery (from $69 \pm 28 \%$ to $29 \pm 9 \%$; mean reduction from baseline $-59 \%$ ). This reduction was present only in the cohort of NENs (differently from what observed in patients affected by other non-neuroendocrine pulmonary cancers, also enrolled in the study); $95 \%$ of surgically treated patients were R0 at histological examination, but no data on longer follow-up or radiological examinations were provided [38].

The 6 prospective and 2 retrospective studies reporting data on NETest after surgical intervention are summarized in Table 3. In conclusion, NETest values between 20 and $40 \%$ can identify minimal residual disease in patients after apparently radical surgery. In this view, patients with negative NETest could need less close follow-up, reducing the number of total body imaging, nuclear or conventional, with clear advantages not only from an economic point of view but also for the lower exposure to radiation [52], in a personalized medicine perspective [53].

\section{Future: Beyond NETest}

In addition to the diagnostic and prognostic role of NETest, the evaluation of tumor transcription may provide additional information. In a study on 20 patients affected by small intestine NETs, a specific subgroup of profibrotic circulating transcripts, the "Fibrosome" of the
NETest was able to predict mesenteric fibrosis in $100 \%$ of cases, even when conventional radiology was negative, providing important information to the surgeon [54]. In this sense, NETest, as other liquid biopsies, may be considered a window on the tumor, providing different types of information. Moreover, the research on NETest is continuing to develop. In 2020, Kidd et al. [55] evaluated the expression of NET-omes and their combinations in a cohort of 88 patients affected by G1 and G2 GEP-NETs, with the aim to identify pathologically relevant -omes for defining of disease status, and to investigate if these elements could provide added prognostic information to the "classical" NETest score. Scores were assessed at baseline and after a median follow-up of 9 months. Four NETomes among those analyzed showed a prognostic value, defined as the correlation between basal levels and outcome (Prognosome: Metastasome, Epigenome, Fibrosome, and NEDome). Then, the authors further investigated the prognostic role of NETest integrated with Prognosome levels. They found that the association at baseline between a low NETest score $(<40)$ and Prognosome levels below the upper limit of normal was an accurate prognostic factor for SD during follow-up (90\%); on the contrary, a high NETest score $(>40 \%)$ associated with Prognosome levels above the upper limit of normal predicted PD within 3 months (100\%). Integrating the 4 -omes with the NETest score (using $40 \%$ as a cut-off) generated an overall prognostic accuracy of $93 \%$, significantly better than the prognostic value of either the NETest alone (70.5\%) or the -omic analysis as a separate approach (69\%) [55].

\section{Conclusions}

\section{Prognostic Assessment by NETest: Ready for Clinical} Application?

Prognostic markers in the field of NENs are lacking and necessary. NETest is the most characterized and validated application of the liquid biopsy to the field of NENs, reported as a key diagnostic advantage, and a promising tool for clinical practice [56]. Beyond its diagnostic value it is also a prognostic tool. In fact, a recent meta-analysis considering data from 6 studies estimated the accuracy of NETest of $84.5-85.5 \%$ in differentiating SD from PD [20]. By this systematic review, we tried to answer specific questions, which can be useful for clinical practice, as summarized in Figure 2. The 2 fields with more available data on the prognostic value of NETest are: the ability of NETest in differentiating SD from PD at baseline and the capacity to predict surgical treatment 


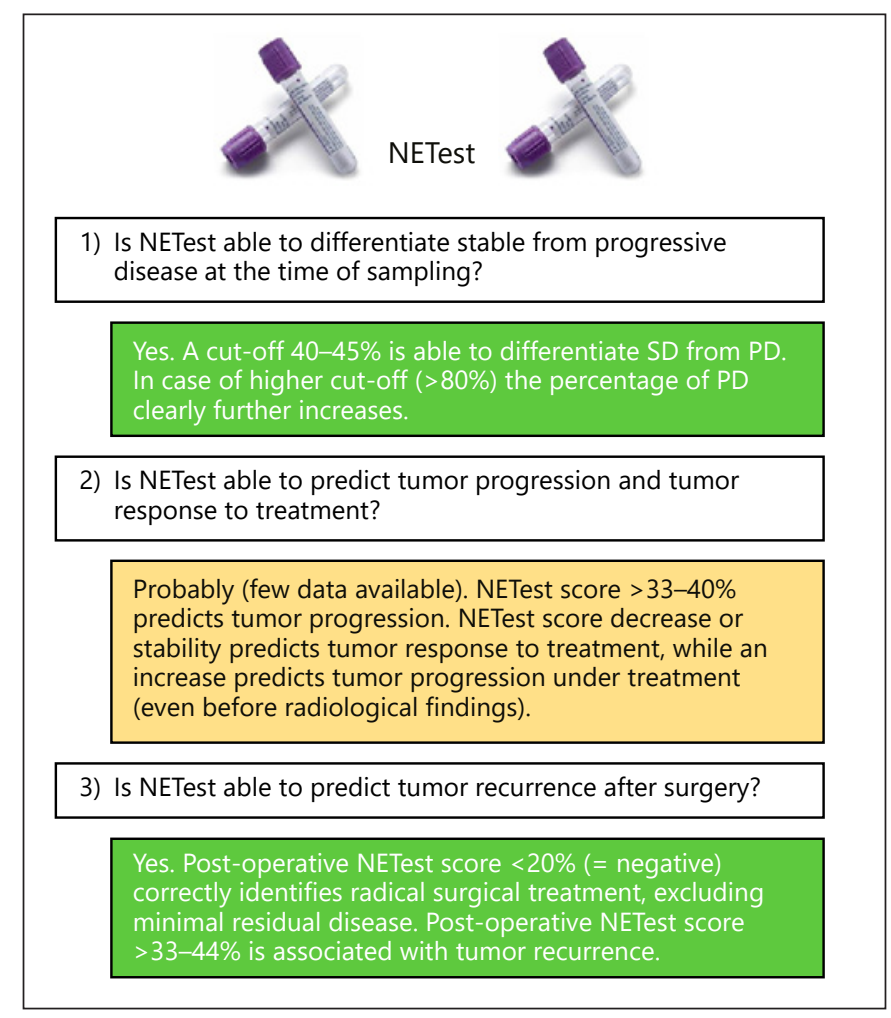

Fig. 2. Possible clinical applications of NETest. Summary of questions and answers debated in the systematic review.

outcome. In particular, high levels of NETest (at least $>40 \%$ ) identify PD. Consequently, NETest could be useful in all situations in which it is important to identify progression, as, for example, in case of new diagnosis of NEN, when it is not possible to establish disease course because previous imaging is clearly unavailable. High baseline NETest levels predict also a subsequent progression, even in case of SSA treatment. These patients should be probably evaluated frequently and treated in a more aggressive way, even if data seem not enough strength for applying this indication to clinical practice.

Finally, given the possibility to assist to a recurrence after an apparently radical surgical procedure, another application of NETest can be the identification of minimal residual disease. Probably, only negative value of NETest $(<20 \%)$, defined as values found in healthy controls [31], correctly identified radical surgical treatment. In this sense, NETest can evaluate patients at high risk of recurrence who could benefit from a more frequent radiological examination or an adjuvant approach. In the authors' opinion, the introduction of NETest in clinical practice should derive from this last application: to identify pa- tients at high risk of recurrence despite the radical surgery, confirmed by no tumor infiltration in the resection margins at histological examination. In this setting, NETest seems not only informative but also able to reduce medical costs through the possibility to reduce the number of morphological and functional imaging during follow-up.

\section{Acknowledgments}

This work has been supported by the Nettare Unit of Sapienza University. We would like to acknowledge all the members: Domenico Alvaro, Emanuela Anastasi, Antonio Angeloni, Oreste Bagni, Caterina Bangrazi, Massimiliano Bassi, Mario Bezzi, Nadia Bulzonetti, Vito Cantisani, Roberto Caronna, Giovanni Casella, Carlo Catalano, Roberta Centello, Enrico Cortesi, Ferdinando D’Ambrosio, Carlo Della Rocca, Adriano De Santis, Cira Di Gioia, Valentina Di Vito, Antongiulio Faggiano, Tiziana Feola, Daniele Gianfrilli, Alfredo Genco, Elisa Giannetta, Franco Iafrate, Andrea M. Isidori, Andrea Lenzi, Paolo Marchetti, Francesca Maccioni, Giulia Puliani, Carla Pandozzi, Franz Sesti, Carola Severi, Silverio Tomao, Vincenzo Tombolini, Federico Venuta, and Monica Verrico.

\section{Statement of Ethics}

An ethics statement is not applicable because this study is based exclusively on published literature.

\section{Conflict of Interest Statement}

The authors have no conflicts of interest to declare.

\section{Funding Sources}

This work was supported by the Ministerial research project PRIN2017Z3N3YC.

\section{Author Contributions}

G.P. and V.D.V. wrote the original draft and performed data collection, data analysis, and interpretation; T.F., F.S., R.C., C.P., and M.G.T. performed data analysis and interpretation; M.V., A.L., and A.M.I. provided the critical revision of the article; E.G. supervised the findings of this work and provided the critical revision of the article; A.F. provided conceptualization and the critical revision of the article.

\section{Data Availability Statement}

Data sharing is not applicable to this article as no datasets were generated or analyzed during the current study. 


\section{References}

1 Oberg K, Krenning E, Sundin A, Bodei L, Kidd M, Tesselaar M, et al. A Delphic consensus assessment: imaging and biomarkers in gastroenteropancreatic neuroendocrine tumor disease management. Endocr Connect. 2016 Sep;5(5):174-87.

2 Yao JC, Hassan M, Phan A, Dagohoy C, Leary C, Mares JE, et al. One hundred years after "carcinoid": epidemiology of and prognostic factors for neuroendocrine tumors in 35,825 cases in the United States. J Clin Oncol. 2008 Jun 20;26(18):3063-72.

3 Oberg K, Couvelard A, Delle Fave G, Gross D, Grossman A, Jensen RT, et al. ENETS consensus guidelines for standard of care in neuroendocrine tumours: biochemical markers. Neuroendocrinology. 2017;105(3):201-11.

4 Marotta V, Zatelli MC, Sciammarella C, Ambrosio MR, Bondanelli M, Colao A, et al. Chromogranin A as circulating marker for diagnosis and management of neuroendocrine neoplasms: more flaws than fame. Endocr Relat Cancer. 2018 Jan;25(1):R11-29.

5 Janson ET, Holmberg L, Stridsberg M, Eriksson B, Theodorsson E, Wilander E, et al. Carcinoid tumors: analysis of prognostic factors and survival in 301 patients from a referral center. Ann Oncol. 1997 Jul;8(7):685-90.

6 Ahmed A, Turner G, King B, Jones L, Culliford D, McCance D, et al. Midgut neuroendocrine tumours with liver metastases: results of the UKINETS study. Endocr Relat Cancer. 2009 Sep;16(3):885-94.

7 Zatelli MC, Torta M, Leon A, Ambrosio MR, Gion M, Tomassetti P, et al. Chromogranin A as a marker of neuroendocrine neoplasia: an Italian Multicenter Study. Endocr Relat Cancer. 2007 Jun; 14(2):473-82.

8 Levinson SS, Miller JJ. Towards a better understanding of heterophile (and the like) antibody interference with modern immunoassays. Clin Chim Acta. 2002 Nov;325(1-2):115.

9 Herrera-Martinez AD, Hofland LJ, Galvez Moreno MA, Castano JP, de Herder WW, Feelders RA. Neuroendocrine neoplasms: current and potential diagnostic, predictive and prognostic markers. Endocr Relat Cancer. 2019 Mar 1;26(3):R157-79.

10 Chan DL, Clarke SJ, Diakos CI, Roach PJ, Bailey DL, Singh S, et al. Prognostic and predictive biomarkers in neuroendocrine tumours. Crit Rev Oncol Hematol. 2017 May;113:26882.

11 Yao JC, Pavel M, Phan AT, Kulke MH, Hoosen S, St Peter J, et al. Chromogranin A and neuron-specific enolase as prognostic markers in patients with advanced pNET treated with everolimus. J Clin Endocrinol Metab. 2011 Dec;96(12):3741-9.

12 Modlin IM, Oberg K, Taylor A, Drozdov I, Bodei L, Kidd M. Neuroendocrine tumor biomarkers: current status and perspectives. Neuroendocrinology. 2014;100(4):265-77.
13 van Adrichem RC, Kamp K, Vandamme T, Peeters M, Feelders RA, de Herder WW. Serum neuron-specific enolase level is an independent predictor of overall survival in patients with gastroenteropancreatic neuroendocrine tumors. Ann Oncol. 2016 Apr;27(4): 746-7.

14 Petrovic M, Bukumiric Z, Zdravkovic V, Mitrovic S, Atkinson HD, Jurisic V. The prognostic significance of the circulating neuroendocrine markers chromogranin A, pro-gastrin-releasing peptide, and neuron-specific enolase in patients with small-cell lung cancer. Med Oncol. 2014 Feb;31(2):823.

15 Korse CM, Taal BG, Vincent A, van Velthuysen ML, Baas P, Buning-Kager JC, et al. Choice of tumour markers in patients with neuroendocrine tumours is dependent on the histological grade. A marker study of chromogranin A, neuron specific enolase, progastrin-releasing peptide and cytokeratin fragments. Eur J Cancer. 2012 Mar;48(5):662-71.

16 Sarraf KM, Belcher E, Raevsky E, Nicholson AG, Goldstraw P, Lim E. Neutrophil/lymphocyte ratio and its association with survival after complete resection in non-small cell lung cancer. J Thorac Cardiovasc Surg. 2009 Feb; 137(2):425-8.

17 Okui M, Yamamichi T, Asakawa A, Harada M, Saito M, Horio H. Prognostic significance of neutrophil-lymphocyte ratios in large cell neuroendocrine carcinoma. Gen Thorac Cardiovasc Surg. 2017 Nov;65(11):633-9.

18 Cao LL, Lu J, Lin JX, Zheng CH, Li P, Xie JW, et al. A novel predictive model based on preoperative blood neutrophil-to-lymphocyte ratio for survival prognosis in patients with gastric neuroendocrine neoplasms. Oncotarget. 2016 Jul 5;7(27):42045-58.

19 Grenader T, Pavel ME, Ruszniewski PB, Ćwikła JB, Phan AT, Raderer M, et al. Prognostic value of the neutrophil/lymphocyte ratio in enteropancreatic neuroendocrine tumors. Anticancer Drugs. 2020 Mar;31(3): 216-22.

20 Oberg K, Califano A, Strosberg JR, Ma S, Pape $\mathrm{U}$, Bodei L, et al. A meta-analysis of the accuracy of a neuroendocrine tumor mRNA genomic biomarker (NETest) in blood. Ann Oncol. 2020 Feb;31(2):202-12.

21 Moher D, Liberati A, Tetzlaff J, Altman DG; PRISMA Group. Preferred reporting items for systematic reviews and meta-analyses: the PRISMA statement. BMJ. 2009 Oct;339(10): b2535-12.

22 Slim K, Nini E, Forestier D, Kwiatkowski F, Panis Y, Chipponi J. Methodological index for non-randomized studies (minors): development and validation of a new instrument. ANZ J Surg. 2003 Sep;73(9):712-6.
23 Kuznetsov HS, Marsh T, Markens BA, Castaño Z, Greene-Colozzi A, Hay SA, et al. Identification of luminal breast cancers that establish a tumor-supportive macroenvironment defined by proangiogenic platelets and bone marrow-derived cells. Cancer Discov. 2012 Dec;2(12):1150-65.

24 Joosse SA, Pantel K. Tumor-educated platelets as liquid biopsy in cancer patients. Cancer Cell. 2015 Nov 9;28(5):552-4.

25 Siravegna G, Marsoni S, Siena S, Bardelli A. Integrating liquid biopsies into the management of cancer. Nat Rev Clin Oncol. 2017 Sep; 14(9):531-48.

26 Allott EH, Geradts J, Sun X, Cohen SM, Zirpoli GR, Khoury T, et al. Intratumoral heterogeneity as a source of discordance in breast cancer biomarker classification. Breast Cancer Res. 2016 Jun 28;18(1):68.

27 De Luca F, Rotunno G, Salvianti F, Galardi F, Pestrin M, Gabellini S, et al. Mutational analysis of single circulating tumor cells by next generation sequencing in metastatic breast cancer. Oncotarget. 2016 May 3;7(18):2610719.

28 Modlin IM, Kidd M, Bodei L, Drozdov I, Aslanian $\mathrm{H}$. The clinical utility of a novel bloodbased multi-transcriptome assay for the diagnosis of neuroendocrine tumors of the gastrointestinal tract. Am J Gastroenterol. 2015 Aug;110(8):1223-32.

29 Peczkowska M, Cwikla J, Kidd M, Lewczuk A, Kolasinska-Cwikla A, Niec D, et al. The clinical utility of circulating neuroendocrine gene transcript analysis in well-differentiated paragangliomas and pheochromocytomas. Eur J Endocrinol. 2017 Feb;176(2):143-57.

30 Kidd M, Modlin IM, Drozdov I, Aslanian H, Bodei L, Matar S, et al. A liquid biopsy for bronchopulmonary/lung carcinoid diagnosis. Oncotarget. 2018 Jan 23;9(6):7182-96.

31 Modlin IM, Kidd M, Malczewska A, Drozdov I, Bodei L, Matar S, et al. The NETest: the clinical utility of multigene blood analysis in the diagnosis and management of neuroendocrine tumors. Endocrinol Metab Clin North Am. 2018 Sep;47(3):485-504.

32 Malczewska A, Kos-Kudła B, Kidd M, Drozdov I, Bodei L, Matar S, et al. The clinical applications of a multigene liquid biopsy (NETest) in neuroendocrine tumors. Adv Med Sci. 2020 Mar;65(1):18-29.

33 Modlin IM, Drozdov I, Kidd M. The identification of gut neuroendocrine tumor disease by multiple synchronous transcript analysis in blood. PLoS One. 2013;8(5):e63364.

34 Kidd M, Drozdov I, Modlin I. Blood and tissue neuroendocrine tumor gene cluster analysis correlate, define hallmarks and predict disease status. Endocr Relat Cancer. 2015 Aug;22(4):561-75. 
35 Modlin IM, Frilling A, Salem RR, Alaimo D, Drymousis P, Wasan HS, et al. Blood measurement of neuroendocrine gene transcripts defines the effectiveness of operative resection and ablation strategies. Surgery. 2016 Jan;159(1):336-47.

36 Cwikla JB, Bodei L, Kolasinska-Cwikla A, Sankowski A, Modlin IM, Kidd M. Circulating transcript analysis (NETest) in GEPNETs treated with somatostatin analogs defines therapy. J Clin Endocrinol Metab. 2015 Nov;100(11):E1437-45.

37 Pavel M, Jann H, Prasad V, Drozdov I, Modlin IM, Kidd M. NET blood transcript analysis defines the crossing of the clinical rubicon: when stable disease becomes progressive. Neuroendocrinology. 2017;104(2):170-82.

38 Filosso PL, Kidd M, Roffinella M, Lewczuk A, Chung KM, Kolasinska-Cwikla A, et al. The utility of blood neuroendocrine gene transcript measurement in the diagnosis of bronchopulmonary neuroendocrine tumours and as a tool to evaluate surgical resection and disease progression. Eur J Cardiothorac Surg. 2018 Mar 1;53(3):631-9.

39 Liu E, Paulson S, Gulati A, Freudman J, Grosh W, Kafer S, et al. Assessment of NETest clinical utility in a U.S. registry-based study. Oncologist. 2019 Jun;24(6):783-90.

40 Malczewska A, Witkowska M, Makulik K, Bocian A, Walter A, Pilch-Kowalczyk J, et al. NETest liquid biopsy is diagnostic of small intestine and pancreatic neuroendocrine tumors and correlates with imaging. Endocr Connect. 2019 Mar 1;8(4):442-53.

41 Malczewska A, Oberg K, Bodei L, Aslanian H, Lewczuk A, Filosso PL, et al. NETest liquid biopsy is diagnostic of lung neuroendocrine tumors and identifies progressive disease. Neuroendocrinology. 2019;108(3):219-31.

42 Malczewska A, Witkowska M, Wojcik-Giertuga M, Kusnierz K, Bocian A, Walter A, et al. Prospective evaluation of the NETest as a liquid biopsy for gastroenteropancreatic and bronchopulmonary neuroendocrine tumours: an ENETS centre of excellence experience. Neuroendocrinology. 2021 Apr 24; 111(4):304-19.
43 van Treijen MJC, Korse CM, van Leeuwaarde RS, Saveur LJ, Vriens MR, Verbeek WHM, et al. Blood transcript profiling for the detection of neuroendocrine tumors: results of a large independent validation study. Front Endocrinol. 2018;9:740.

44 Bodei L, Kidd M, Modlin IM, Severi S, Drozdov I, Nicolini S, et al. Measurement of circulating transcripts and gene cluster analysis predicts and defines therapeutic efficacy of peptide receptor radionuclide therapy (PRRT) in neuroendocrine tumors. Eur J Nucl Med Mol Imaging. 2016 May;43(5): 839-51.

45 van Treijen MJC, van der Zee D, Heeres BC, Staal FCR, Vriens MR, Saveur LJ, et al. Blood molecular genomic analysis predicts the disease course of GEP NET patients: a validation study of the predictive value of the NETest(R). Neuroendocrinology. 2021 Jun 3;111(6):58698.

46 Bodei L, Kidd MS, Singh A, van der Zwan WA, Severi S, Drozdov IA, et al. PRRT neuroendocrine tumor response monitored using circulating transcript analysis: the NETest. Eur J Nucl Med Mol Imaging. 2020 Apr;47(4): 895-906.

47 Laskaratos FM, Liu M, Malczewska A, Ogunbiyi O, Watkins J, Luong TV, et al. Evaluation of circulating transcript analysis (NETest) in small intestinal neuroendocrine neoplasms after surgical resection. Endocrine. 2020 Aug; 69(2):430-40.

48 Frilling A, Clift AK, Frampton AE, Bomanji J, Kaemmerer D, Al-Nahhas A, et al. A combination of surgery, theranostics, and liquid biopsy - a personalised oncologic approach to treatment of patients with advanced metastatic neuroendocrine neoplasms. Int J Med Sci. 2021;18(10):2166-75.

49 Genc CG, Jilesen APJ, Nieveen van Dijkum EJM, Klumpen HJ, van Eijck CHJ, Drozdov I, et al. Measurement of circulating transcript levels (NETest) to detect disease recurrence and improve follow-up after curative surgical resection of well-differentiated pancreatic neuroendocrine tumors. J Surg Oncol. 2018 Jul;118(1):37-48.
50 Partelli S, Andreasi V, Muffatti F, Schiavo Lena M, Falconi M. Circulating neuroendocrine gene transcripts (NETest): a postoperative strategy for early identification of the efficacy of radical surgery for pancreatic neuroendocrine tumors. Ann Surg Oncol. 2020 Oct; 27(10):3928-36.

51 Malczewska A, Procner A, Walter A, Kusnierz K, Zajecki W, Aslanian H, et al. The NETest liquid biopsy is diagnostic for gastric neuroendocrine tumors: observations on the blood-based identification of microscopic and macroscopic residual diseaseOK. BMC Gastroenterol. 2020 Jul 23;20(1):235.

52 Modlin IM, Kidd M, Oberg K, Falconi M, Filosso PL, Frilling A, et al. Early identification of residual disease after neuroendocrine tumor resection using a liquid biopsy multigenomic mRNA signature (NETest). Ann Surg Oncol. 2021 May 18. Epub ahead of print.

53 Rinke A, Auernhammer CJ, Bodei L, Kidd M, Krug S, Lawlor R, et al. Treatment of advanced gastroenteropancreatic neuroendocrine neoplasia, are we on the way to personalised medicine? Gut. 2021 Mar 10;70:176881.

54 Laskaratos FM, Mandair D, Hall A, Alexander S, von Stempel C, Bretherton J, et al. Clinicopathological correlations of mesenteric fibrosis and evaluation of a novel biomarker for fibrosis detection in small bowel neuroendocrine neoplasms. Endocrine. 2020 Mar;67(3): $718-26$.

55 Kidd M, Kitz A, Drozdov I, Modlin I. Neuroendocrine tumor omic gene cluster analysis amplifies the prognostic accuracy of the NETest. Neuroendocrinology. 2021 May 11; 111(5):490-504.

56 Caplin ME, Ratnayake GM. Diagnostic and therapeutic advances in neuroendocrine tumours. Nat Rev Endocrinol. 2021 Feb;17(2): 81-2. 